\title{
Large investor trading impacts on volatility
}

\author{
Pierre-Louis Lions $^{\text {a,b,* }}$, Jean-Michel Lasry ${ }^{\mathrm{c}}$ \\ a CEREMADE - UMR C.N.R.S. 7534, Université Paris 9 - Dauphine, Place du Maréchal de Lattre de Tassigny, 75775 Paris Cedex 16, France \\ ${ }^{\mathrm{b}}$ Collège de France, 11, place Marcelin Berthelot, 75005 Paris, France \\ c CALYON, 9, Quai du Président Paul Doumer, 92920 Paris, La défense cedex, France
}

Received 21 November 2005; accepted 21 December 2005

Available online 14 November 2006

\begin{abstract}
We begin with this paper a series devoted to a tentative model for the influence of hedging on the dynamics of an asset. We study here the case of a "large" investor and solve two problems in the context of such a model namely the question of the fair value (or liquidative value) of a "large" position and the question of pricing or hedging an option. In order to do so, we use a utility maximization approach and some new results in stochastic control theory.

(C) 2006 Published by Elsevier Masson SAS.
\end{abstract}

\section{Introduction}

This paper is the first of a series devoted to the effect of reconciling the classical Black-Scholes theory of option pricing and hedging with various phenomena observed in the markets such as the influence of trading and hedging on the dynamics of an asset. Assuming, for simplicity, that the price of an asset is modified by trading and hedging through a linear "elastic" law, we shall prove that the optimal hedging strategy derived from a utility maximization principle impacts upon the volatility of the asset.

More precisely, we shall consider here the case of a "large" investor whose trading influences the dynamics of an asset price. And we study two related problems

(i) what is the "liquidation" value of a position? As is well known, this value that might be called the fair value is not simply derived from the market price (as is natural, since selling a sizable number will immediately make the price of the asset go down...). Our approach will, in some sense, allow to reconcile the notion of fair value with market prices (mark to market). And we shall also see the impact on the volatility;

(ii) how is the fundamental F. Black and M. Scholes [5], R. Merton [14] theory for option pricing and hedging modified by our basic assumption on the price dynamics?

At this stage, and before describing more precisely our main results, we wish to mention some previous related works concerned with some "phenomenological" models of the influence factor we are investigating and we refer to

\footnotetext{
* Corresponding author.

E-mail address: lions@ ceremade.dauphine.fr(P.-L. Lions).
} 
A.S. Kyle [10], K. Back [2], K. Back, C.H. Cao and G. Willard [3], H. Föllmer [8], R. Frey and A. Stremme [9]. In fact, we discovered these references after introducing and testing closely related phenomenological models for some particular type of financial products. And, when we observed some practical relevance, our goal was to study this type of phenomenon and derive rigorously from first principles such models or rigorous models related to those phenomenological ones.

Let us also point out that our second paper in this series [11] is devoted to an attempt of a rigorous theory incorporating the price influences of a "large" number of "small" (compared to the total market activity) investors/traders in a self-consistent way and leading to a tentative theory for the formation of volatility.

Finally, we wish to emphasize the fact that, although we modify drastically the basic hypothesis on the asset price dynamics (as an exogenous diffusion process a priori determined), the classical Black-Scholes theory is quite resilient and our results will clearly show that its basic structure remains the same even though the precise details are indeed completely changed.

We now conclude this long introduction by introducing our main notation and assumption, and explaining more precisely the problems we solve below. We begin with a simple model due to A.S. Kyle [10] (see also K. Back [2], K. Back, C.H. Cao and G. Willard [3]) for the dynamics of a single underlying asset which we assume to be given by

$$
\mathrm{d} S_{t}=\sigma\left(S_{t}\right) \mathrm{d} B_{t}-k u_{t} \mathrm{~d} t,
$$

where $\left(\Omega, F, F_{t}, P, B_{t}\right)$ is a standard Wiener space ( $B_{t}$ is a standard Brownian motion), $\sigma$ is a given function (assumed, for instance, to be bounded from above and from below away from 0 , and Lipschitz on $\mathbb{R}$ ) that corresponds to some a priori exogenous volatility and $k$ is a positive parameter. The term $\left(u_{t} \mathrm{~d} t\right)$ corresponds to the rate at which a single "large" investor sells or buys the asset, thus modifying instantaneously the price $S_{T}$ by a simple linear law (of offer and demand). The quantity of the asset owned by this investor is then denoted by $\alpha_{t}$ and we thus have

$$
\mathrm{d} \alpha_{t}=-u_{t} \mathrm{~d} t .
$$

The corresponding wealth in the classical Black-Scholes theory is always taken to be $\alpha_{t} S_{t}$ i.e. the amount of cash obtained from liquidating the position. This is of course meaningless if $k \neq 0$ and we thus cannot define a priori the wealth (it is in fact precisely one of the issues we consider and solve below...). We thus simply consider the cash $C_{t}$ used in the trading of $S_{t}$ namely

$$
\mathrm{d} C_{t}=u_{t} S_{t} \mathrm{~d} t .
$$

Observe that, in order to simplify both the presentation and the notation, we do not incorporate any interest rate (taken to be $0 \ldots)$.

We may now formulate our first problem: let $T>0$, given a position $\alpha$ at time $t$, what is the "maximal" amount of cash we may generate if we request that $\alpha_{T}=0$. In order to formulate precisely this problem that corresponds to a notion of fair value or liquidative value, we use a utility function maximization approach and we consider the following optimal stochastic control problem

$$
V=\sup _{u \in \mathcal{U}}\left\{E\left[U\left(C_{T}\right) \mid C_{t}=C, S_{t}=S, \alpha_{t}=\alpha\right]\right\},
$$

where $\mathcal{U}=\left\{u\right.$ adapted, $\left.E\left[\int_{t}^{\mathrm{T}}\left|u_{s}\right| \mathrm{d} s\right]<\infty \mid \alpha_{T}=0\right\}, t<T, V=V(C, S, \alpha, t) \in(-\infty,+\infty]$ and $U$ is a given utility function that we assume, in order to simplify the presentation (although we shall consider more general ones below), to be $C^{2}$ on $\mathbb{R}$, strictly increasing on $\mathbb{R}$ and $U^{\prime \prime}(z)<0$ for all $z \in \mathbb{R}$. A typical example is the exponential utility namely

$$
U(z)=U_{\lambda}(z)=1-\exp (-\lambda z) \text { for all } z \in \mathbb{R},
$$

where $\lambda>0$ is the absolute risk aversion.

In Section 2 below, we completely solve this problem and we consider as well extensions of it allowing for some drift terms, or relaxing the final target constraint namely $\alpha_{T}=0$, or replacing (1) by

$$
\frac{\mathrm{d} S_{t}}{S_{t}}=\sigma\left(S_{t}\right) \mathrm{d} B_{t}-k u_{t} \mathrm{~d} t .
$$

As we shall see, we shall deduce the following explicit "fair" values

$$
P=\alpha S-k \frac{\alpha^{2}}{2}
$$


in the case of (1), and

$$
P=\frac{1-\mathrm{e}^{-k \alpha}}{\alpha} S
$$

in the case of $\left(1^{\prime}\right)$.

Next, in Section 3, we study the issue of option pricing in such a context namely we replace (4) by

$$
V=\sup _{u \in \mathcal{U}}\left\{E\left[U\left(C_{T}-\Phi\left(S_{T}\right)\right) \mid C_{t}=C, S_{t}=S, \alpha_{t}=\alpha\right]\right\}
$$

where $\Phi$ is the pay-off of a European option. And we shall also consider the case when $k$ is small $(k=\epsilon)$ or, more interestingly, when $\Phi$ is replaced by $\epsilon \Phi$ and $U$ by $U(\dot{\epsilon})$, and then we compute the corrections to the classical BlackScholes theory. Let us also mention that we also consider in that section the more natural situation when we do not require $\alpha_{T}$ to vanish.

As we shall see, both in Sections 2 and 3, not only do we solve the above stochastic control problems but we deduce, through the optimal strategy, the resulting price dynamics. And, as we shall explain, this class of stochastic control problems is a rather new class of singular stochastic control problems which cannot be handled by the classical methods (e.g. Hamilton-Jacobi-Bellman equations) and we have to rely upon the theory we built in [12] in order to incorporate such problems. However, for the "simple" problems above, it is possible to make a direct proof that we shall present, although, this argument uses some tricks whose explanation is a direct consequence of [12]. At this stage, we simply announce that the resulting price dynamics correspond to a modified volatility!

Finally, in Section 4, we briefly mention some possible extensions to an arbitrary number of assets, or more general "influence" models, or models with interest rates....

After this series of works was completed, we became aware of some independent works by M. Avellaneda and M.D. Lipkin [1] and by G. Lasserre [13] that are somewhat related to the topics addressed here.

\section{Fair values}

We begin with the problem (4) or with a more general version of it where we relax the final target constraint namely $\alpha_{T}=0$ :

$$
V=\sup _{u}\left\{E\left[U\left(C_{T}-\frac{W}{2} \alpha_{T}^{2}\right) \mid C_{t}=C, S_{t}=S, \alpha_{t}=\alpha\right]\right\} .
$$

At least formally, we recover the problem (4) letting the positive (weight) parameter $W$ go to $+\infty$. And we first consider the case when $\sigma(S)$ is a constant: $\sigma(S)=\sigma>0$.

As we mentioned in the introduction, this type of stochastic control problems cannot be handled by classical methods such as, e.g., the corresponding Hamilton-Jacobi-Bellman equation (HJB in short) - and we refer the reader to the monographs by W.H. Fleming and H.M. Soner [7], M. Bardi and I. Capuzzo-Dolcetta [4] for a presentation of the classical methods. Indeed, at least formally, the dynamic programming principle "leads" to the following (formal) HJB equation

$$
\frac{\partial V}{\partial t}+\frac{\sigma^{2}}{2} \frac{\partial^{2} V}{\partial S^{2}}+\sup _{u \in \mathbb{R}}\left\{u\left(S \frac{\partial V}{\partial C}-k \frac{\partial V}{\partial S}-\frac{\partial V}{\partial \alpha}\right)\right\}=0
$$

with $\left.V\right|_{t=T}=U\left(C-\frac{W}{2} \alpha^{2}\right)$, and this equation does not contain enough information in order to characterize $V$ since the supremum in $u$ is always infinite unless we have

$$
S \frac{\partial V}{\partial C}-k \frac{\partial V}{\partial S}-\frac{\partial V}{\partial \alpha}=0 .
$$

This equation means, at least formally, that $V$ should be invariant by the flow

$$
\Psi_{\mu}(C, S, \alpha)=\left(C+\mu S-k \frac{\mu^{2}}{2}, S-k \mu, \alpha-\mu\right) \quad(\mu \in \mathbb{R})
$$

which is nothing else than the integral flow associated to

$$
\dot{S}=-k, \quad \dot{\alpha}=-1, \quad \dot{C}=S
$$


(or (1)-(3) with $\sigma \equiv 0$ and $u \equiv 1$ ). Observe also that $V$ at time $T$ (namely $V=V\left(C-\frac{W}{2} \alpha^{2}\right)$ ) is not invariant by this flow!

This type of stochastic control problems is completely solved in J.-M. Lasry and P.-L. Lions [12] where it is shown that $V$ is indeed invariant by the flow (9) for $t<T$ (with a terminal boundary layer at $t=T$ ) and that it is possible to write for $V$ a reduced stochastic control problem (in some vague sense, a stochastic control problem on the quotient space induced by the orbits of the flow) which can then be solved by the classical methods of stochastic control theory.

Instead of applying blindly this general theory, we extract from it some crucial facts and present some direct arguments (let us emphasize however that this is possible here only because we carefully selected problems that are simple enough...). Indeed, the "expected" invariance of $V$ by the flow means that $V$ should only depend upon two reduced variables and a natural choice, in view of (9), is

$$
P=C+\alpha S-k \frac{\alpha^{2}}{2}, \quad X=S-k \alpha .
$$

As we shall see later on, $P$ corresponds to the total wealth (or cash) and $X$ is the price of the asset when one takes $\alpha$ instantaneously to 0 . Let us mention at this stage that similar choices of "reduced" variables were introduced previously by K. Back, C.H. Cao and G.A. Willard [3].

We thus reformulate (1)-(3), (4) and (8) with this new set of variables: we obviously find

$$
\mathrm{d} X_{t}=\sigma \mathrm{d} B_{t}
$$

(here we use the fact that $\sigma$ is a constant...), and

$$
\begin{aligned}
\mathrm{d} P_{t} & =\mathrm{d} C_{t}+\alpha_{t} \mathrm{~d} S_{t}+S_{t} \mathrm{~d} \alpha_{t}-k \alpha_{t} \mathrm{~d} \alpha_{t} \\
& =u_{t} S_{t} \mathrm{~d} t+\alpha_{t}\left(\sigma \mathrm{d} B_{t}-k u_{t} \mathrm{~d} t\right)-S_{t} u_{t} \mathrm{~d} t+k u_{t} \alpha_{t} \mathrm{~d} t
\end{aligned}
$$

or

$$
\mathrm{d} P_{t}=\alpha_{t} \sigma \mathrm{d} B_{t}=\alpha_{t} \mathrm{~d} X_{t},
$$

i.e. the classical evolution of wealth in terms of the hedge $\alpha_{t}$.

Using the invariance and the constraint $\alpha_{T}=0$, we then deduce that (4) is "equivalent" for $t<T$ to

$$
V(P, X, t)=\sup \left\{E\left[U\left(P_{T}\right) \mid P_{t}=P, X_{t}=X\right] \mid \alpha_{t} \text { adapted }\right\} .
$$

In the case of (8), we need to introduce

$$
\sup _{\mu \in \mathbb{R}} U\left(C_{\mu}-\frac{W}{2} \alpha_{\mu}^{2}\right)=U\left(\sup _{\mu \in \mathbb{R}}\left(C_{\mu}-\frac{W}{2} \alpha_{\mu}^{2}\right)\right),
$$

where $C_{\mu}=C+\mu S-k \frac{\mu^{2}}{2}, \alpha_{\mu}=\alpha-\mu$. We observe that

$$
\begin{aligned}
\sup _{\mu \in \mathbb{R}}\left(C_{\mu}-\frac{W}{2} \alpha_{\mu}^{2}\right) & =C-\frac{W}{2} \alpha^{2}+\frac{1}{2} \frac{(S+\alpha W)^{2}}{k+W} \\
& =P-\alpha S+\frac{k \alpha^{2}}{2}-\frac{W}{2} \alpha^{2}+\frac{1}{2} \frac{\left(X+\alpha(k+W)^{2}\right.}{k+W} \\
& =P-\alpha X-\frac{k+W}{2} \alpha^{2}+\frac{1}{2} \frac{(X+\alpha(k+W))^{2}}{k+W} \\
& =P+\frac{1}{2} \frac{X^{2}}{k+W} .
\end{aligned}
$$

And we claim that (8) is "equivalent" for $t<T$ to

$$
V(P, X, t)=\sup \left\{E\left[U\left(P_{T}+\frac{1}{2(k+W)} X_{T}^{2}\right)\right]\left|P_{t}=P, X_{t}=X\right| \alpha_{t} \text { adapted }\right\} .
$$

From a rigorous mathematical viewpoint, the equivalence is a simple application of our results in [12] and we cannot repeat the detailed proof here but it is possible to make a convincing argument for the above modification of the 
terminal reward function $U\left(C-\frac{W}{2} \alpha^{2}\right)$ : indeed, as soon as $t$ is strictly smaller than $T$ (and very close to $T, t=T-\epsilon$, we may use as possible controls $u_{\epsilon} \equiv \frac{\mu}{\epsilon}$ on $[T-\epsilon, T]$ in which case we deduce easily that $\alpha_{T}=\alpha-\mu, S_{T} \approx S-k \mu$ and $C_{T} \approx C+\mu S-k \frac{\mu^{2}}{2}$. Therefore, we may "jump instantanously" (or almost) along the orbit of the flow and thus we should only consider the supremum along the orbit of the flow of the final reward function which is precisely the quantity we computed above.

And we are led to the

Theorem 2.1. Assume that $\sigma$ is constant.

(1) The value function $V$ given by (13) satisfies

$$
V=U\left(C+\alpha S-k \frac{\alpha^{2}}{2}\right) \text { for all } C, S \in \mathbb{R} .
$$

(2) The value function $V$ given by (14) satisfies

$$
V=U\left(C+\frac{S^{2}}{2(k+W)}+\frac{W}{k+W} \alpha S-\frac{k W}{k+W}-(T-t) \frac{\sigma^{2}}{2(k+W)}\right),
$$

the associated optimal strategy $\alpha_{t}$ satisfies

$$
\mathrm{d} \alpha_{t}=-\frac{\sigma}{k+W} \mathrm{~d} B_{t}
$$

and the induced price dynamics are given by

$$
\mathrm{d} S_{t}=\sigma \frac{W}{k+W} \mathrm{~d} B_{t}
$$

Remark 2.1. Part (1) means that the fair value or liquidative value is given by $\alpha S-k \frac{\alpha^{2}}{2}$. Obviously, when $k=0$, we recover the usual "market" value $\alpha S$. As we shall see below, this is in fact the case for an arbitrary volatility $\sigma$ (we may already observe that it is independent of the constant $\sigma$ ).

Remark 2.2. All the above explicit formulas are independent of the utility function.

Remark 2.3. It is not difficult to extend the above result to the case when we add a drift term to (1) i.e.

$$
\mathrm{d} S_{t}=\sigma \mathrm{d} B_{t}+b \mathrm{~d} t-k u_{t} \mathrm{~d} t
$$

(where $b$ is a constant). We leave such easy extensions to the reader.

Conclusion of the proof of Theorem 2.1. There only remains to show that the value function $V$ given by (14) satisfies

$$
V=U\left(P+\frac{X^{2}}{2(k+W)}-(T-t) \frac{\sigma^{2}}{2(k+W)}\right)
$$

with an optimal strategy $\alpha$ given by: $\alpha=-\frac{X}{k+W}$, since (16)-(18) then follow from (10), (1) and (2). Finally, (15) may be, for instance, deduced from (16) upon letting $W$ go to $+\infty$ (or directly from (13) which yields easily, since $U$ is strictly concave, that $V=U(P))$.

Finally, in order to prove the above claims, we may simply use HJB equations which, here, take the following form

$$
\frac{\partial V}{\partial t}+\frac{1}{2} \sigma^{2} \frac{\partial^{2} V}{\partial X^{2}}+\sup _{\alpha \in \mathbb{R}}\left\{\sigma^{2} \frac{\alpha^{2}}{2} \frac{\partial^{2} V}{\partial P^{2}}+\sigma^{2} \alpha \frac{\partial^{2} V}{\partial X \partial P}\right\}=0
$$

with $\left.V\right|_{t=T}=U\left(P+\frac{X^{2}}{2(k+W)}\right)$. The above claims then follow from a simple verification that we skip.

We now check that the formula (15) is in fact valid for an arbitrary $\sigma(S)$. 
Theorem 2.2. The value function defined by (4) is given by (15).

Proof. The reduction made before Theorem 2.2 remains valid provided we replace $\sigma$ by $\sigma\left(S_{t}\right)=\sigma\left(X_{t}+k \alpha_{t}\right)$. Then, $V$ solves the following HJB equation (one may also argue directly using the concavity of the utility function $U$ ).

$$
\frac{\partial V}{\partial t}+\sup _{\alpha \in \mathbb{R}}\left\{\frac{1}{2} \sigma^{2}(X+k \alpha)\left(\frac{\partial^{2} V}{\partial X^{2}}+\alpha^{2} \frac{\partial^{2} V}{\partial P^{2}}+\alpha \frac{\partial^{2} V}{\partial X \partial P}\right)\right\}=0
$$

and $\left.V\right|_{t=T}=U(P)$. Obviously, we have: $V=U(P)$ and (15) is shown.

Remark 2.4. In the results above ( and in those of the next section) one obtains an optimal hedging strategy which is not of bounded variation (in fact, in Section 3, we shall see some example where $\alpha_{t}$ may even have no differential properties whatsoever...). In other words, there is no possible interpretation in terms of an optimal $u_{t}$ (with $\left.\mathrm{d} \alpha_{t}=u_{t} \mathrm{~d} t\right)$ ! This is a consequence of the singular nature of the optimal stochastic control problems we are solving, which transforms a state variable into a control. A related, even simpler, example is given by

$$
\left\{\begin{array}{l}
\mathrm{d} x_{t}=\mathrm{d} W_{t_{1}}, x_{0}=x \in \mathbb{R} ; \quad \mathrm{d} y_{y}=u_{t} \mathrm{~d} t, \quad y_{0}=y \in \mathbb{R} \\
\sup \left\{E\left[F\left(x_{T}, y_{T}\right)\right] \mid u_{t} \text { adapted }, u_{t} \in L^{1}(0, T ; \mathbb{R})\right\}=V(x, y, T),
\end{array}\right.
$$

where $F$ is, say, continuous and bounded from above on $\mathbb{R}^{2}$. As is easily checked, the problem reduces to the following one for $T>0$

$$
\begin{aligned}
V(x, y, T)=V(x, T) & =\sup \left\{E\left[F\left(x_{T}, y_{T}\right)\right] \mid y_{t} \text { adapted, } y_{T} \in L^{1}(0, T ; \mathbb{R})\right\} \\
& =E\left[\sup _{y \in \mathbb{R}} F\left(x_{T}, y\right)\right] .
\end{aligned}
$$

The state variable $y$ has become a control and $y_{t}$ is optimal if $y_{t}$ is a maximum point of $F\left(x_{t}, y\right)$ over $\mathbb{R}$. For instance, if $F(x, y)=-|x| \frac{y^{2}}{2}+x y-\frac{|x|}{2}$, the optimal feedback is given by $y=\operatorname{sign}(x)$ and thus $y_{t}=\operatorname{sign}\left(x+W_{t}\right)$ which has no differentiability properties...!

We conclude this section by considering the case when (1) is replaced by $\left(1^{\prime}\right)$. Then, the value function $V$ (defined by (4) is invariant by the flow

$$
\begin{aligned}
& \Psi_{\mu}(C, S, \alpha)=\left(C+\frac{1-\mathrm{e}^{-k \mu}}{k} S, \mathrm{e}^{-k \mu} S, \alpha-\mu\right), \\
& P=C+\frac{1-\mathrm{e}^{-k \alpha}}{k} S, \quad X=\mathrm{e}^{-k \alpha} S .
\end{aligned}
$$

And we now find

$$
\mathrm{d} X_{t}=\mathrm{e}^{-k \alpha_{t}} \mathrm{~d} S_{t}-k \mathrm{e}^{-k \alpha_{t}} S_{t} \mathrm{~d} \alpha_{t}
$$

or

$$
\frac{\mathrm{d} X_{t}}{X_{t}}=\sigma\left(\mathrm{e}^{k \alpha_{t}} X_{t}\right) \mathrm{d} B_{t},
$$

and

$$
\begin{aligned}
\mathrm{d} P_{t} & =u_{t} S_{t} \mathrm{~d} t+\frac{1-\mathrm{e}^{-k \alpha_{t}}}{k} \mathrm{~d} S_{t}+\mathrm{e}^{-k \alpha_{t}} S_{t} \mathrm{~d} \alpha_{t} \\
& =\frac{1-\mathrm{e}^{-k \alpha_{t}}}{k}\left(\sigma\left(\mathrm{e}^{k \alpha_{t}} X_{t}\right) S_{t} \mathrm{~d} B_{t}-k u_{t} S_{t} \mathrm{~d} t\right)+u_{t} S_{t}\left(1-\mathrm{e}^{-k \alpha_{t}}\right) \mathrm{d} t
\end{aligned}
$$

or

$$
\mathrm{d} P_{t}=\frac{\mathrm{e}^{k \alpha_{t}}-1}{k} X_{t} \sigma\left(\mathrm{e}^{k \alpha_{t}} X_{t}\right) \mathrm{d} B_{t}=\frac{\mathrm{e}^{k \alpha_{t}}-1}{k} \mathrm{~d} X_{t} .
$$

Once more, we come back to the "usual" dynamics for wealth except that the "hedge" is now given by $\left(\mathrm{e}^{k \alpha}-1\right) / k$ (instead of $\alpha_{t} \ldots$ ). And, $V$ is still given by (13). We then have the 
Theorem 2.3. In the case of the dynamics $\left(1^{\prime}\right)$, the value function defined by (4) is given by

$$
V=U\left(C+\frac{1-\mathrm{e}^{-k \alpha}}{k} S\right)
$$

Remark 2.5. The analogues of Remarks $2.1-2.3$ are valid in the case of dynamics given by $\left(1^{\prime}\right)$.

The proof of Theorem 2.3 is similar to those made before and we skip it (observe that $V=U(P) \ldots$ ).

\section{Option pricing}

We now turn to the issue of option pricing and hedging under the dynamics (1) (or $\left(1^{\prime}\right)$ ). We thus consider the stochastic control problem (7) where $\Phi$ is the pay-off of the option. In order to avoid technical considerations, we assume that $\Phi$ is uniformly continuous on $\mathbb{R}$ (although discontinuities could be allowed...).

We may still follow the reduction performed in Section 2, introducing $P_{t}=C_{t}+\alpha_{t} S_{t}-k \alpha_{t}^{2} / 2, X_{t}=S_{t}-k \alpha_{t}$, and exactly as in the preceding section, we find that we have

$$
V(P, X, t)=\sup _{\alpha_{t} \text { adapted }} E\left[U\left(P_{T}-\Phi\left(X_{T}\right)\right) \mid P_{t}=P, X_{t}=X\right]
$$

with

$$
\left\{\begin{array}{l}
\mathrm{d} X_{s}=\sigma\left(X_{s}+k \alpha_{s}\right) \mathrm{d} B_{s} \\
\mathrm{~d} P_{s}=\alpha_{s} \sigma\left(X_{s}+k \alpha_{s}\right) \mathrm{d} B_{s}=\alpha_{s} \mathrm{~d} X_{s} .
\end{array}\right.
$$

It is worth observing that $P_{t}$ is precisely the total wealth corresponding to the addition of the cash $C_{t}$ and the value of the quantity $\alpha_{t}$ of the asset whose price is $S_{t}$ (namely $\alpha_{t} S_{t}-k \alpha_{t}^{2} / 2$ as shown in Section 2). Then, as in G. Constantinides and Th. Zariphopoulou [6] (see also M. Musiela and Th. Zariphopoulou [15]...), we define the indifference price $\pi$ of the option by the relationship

$$
V=U(P-\pi)=U\left(C+\alpha S-k \frac{\alpha^{2}}{2}-\pi\right)
$$

since $U(P)$ is the value function corresponding to $\Phi \equiv 0$ (see Section 2). And the hedge is given by the optimal control (in (21)) provided it exists....

At this stage it is worth explaining the role of the somewhat unnatural constraint contained in the definition of the class $\mathcal{U}$ of admissible controls namely $\alpha_{T}=0$. First of all, if we consider the cash at time $T$ namely $C_{T}$ (as in (7)), it is obvious that it does not amount to the total wealth unless $\alpha_{T}=0$. Therefore, if we wish to suppress the constraint $\alpha_{T}=0$, we may do so provided we replace in (7) $C_{T}$ by the effective total wealth at time T namely $C_{T}+\alpha_{T} S_{T}-k \alpha_{T}^{2} / 2$ since we now know from Section 2 that $\alpha_{T} S_{T}-k \alpha_{T}^{2} / 2$ is precisely the "fair" or "liquidative" value corresponding to owning $\alpha_{T}$ of the asset whose price is $S_{T}$. In addition, a fair contract should be written in terms of $\Phi\left(X_{T}\right)=\Phi\left(S_{T}-k \alpha_{T}\right)$ and not of $\Phi\left(S_{T}\right)$ (otherwise the trader could "push" instantaneously just before $T$ to a price corresponding to the minimum value of $\Phi$ i.e. 0 for a call option, an obviously unfair behavior!). But, if the option is written as $\Phi\left(S_{T}-k \alpha_{T}\right)$, we obtain a fair contract in which the trader has no interest in trying to manipulate the price since we have for all $\mu \in \mathbb{R}$

$$
S_{T}-k \mu-k\left(\alpha_{T}-\mu\right)=S_{T}-k \alpha_{T} .
$$

We are thus led to the utility maximization problem (21). In other words, relaxing the terminal constraint $\left(\alpha_{T}=0\right)$ is possible provided one defines a fair contract (as discussed above) in which case the two problems are in fact equivalent!

Our first result is the following

Theorem 3.1. We assume that the utility function is of exponential type (5). Then

$$
V=U\left(C+\alpha S-k \frac{\alpha^{2}}{2}-\pi(S-k \alpha, t)\right),
$$


where the indifference price $\pi$ is the unique viscosity solution (uniformly continuous in $x$, uniformly in $t \leqslant T$ bounded) of the following HJB equation

$$
\frac{\partial \pi}{\partial t}+\frac{1}{2} \inf _{\alpha \in \mathbb{R}}\left\{\sigma^{2}(X+k \alpha)\left[\frac{\partial^{2} \pi}{\partial X^{2}}+\lambda\left(\alpha-\frac{\partial \pi}{\partial X}\right)^{2}\right]\right\}=0 \quad \text { for } X \in \mathbb{R}, t<T,
$$

such that

$$
\left.\pi\right|_{t=T}=\Phi(X) .
$$

Corollary 3.1. If $\sigma$ is independent of $S$, the indifference price reduces to the Black-Scholes price

$$
\pi(X, t)=E\left[\Phi\left(X_{T}\right) \mid X_{t}=X\right]=E\left[\Phi\left(X+\sigma\left(W_{T}-W_{t}\right)\right)\right]
$$

and the optimal strategy is unique and given by $\alpha=\frac{\partial \pi}{\partial X}(X, t)$; hence, the induced dynamics o the price $S_{t}$ of the asset become

$$
\mathrm{d} S_{t}=\sigma\left(1+k \frac{\partial^{2} \pi}{\partial X^{2}}\left(X_{t}, t\right)\right) \mathrm{d} B_{t}, \quad \mathrm{~d} X_{t}=\sigma \mathrm{d} B_{t} .
$$

In addition, the above facts are true for any utility function.

\section{Corollary 3.2.}

(1) As $\lambda$ goes to $0_{+}$(if $k>0$ ), the indifference price $\pi$ goes (uniformly on compact sets) to the unique viscosity solution of

$$
\frac{\partial \pi}{\partial t}+\frac{1}{2} \inf _{y \in \mathbb{R}}\left\{\sigma^{2}(y) \frac{\partial^{2} \pi}{\partial X^{2}}\right\}=0 \quad \text { for } X \in \mathbb{R}, t<T,
$$

satisfying (26);

(2) As $\lambda$ goes to $+\infty$, the indifference price $\pi$ goes (uniformly on compact sets) to the unique viscosity solution of

$$
\frac{\partial \pi}{\partial t}+\frac{1}{2} \sigma^{2}\left(x+k \frac{\partial \pi}{\partial X}\right) \frac{\partial^{2} \pi}{\partial X^{2}}=0 \quad \text { for } X \in \mathbb{R}, t<T,
$$

satisfying (26).

Remark 3.1. Obviously, when $k=0$, we recover the usual Black-Scholes theory for the price and the hedge since the optimal strategy is then simply given by the unique infimum in $\alpha$ (in (25)) namely $\alpha=\partial \pi / \partial X$. We shall come back below to the expansion (correction) as $k$ goes to $0_{+}$.

Remark 3.2. In general, the existence (and meaning) of an optimal hedge may be delicate since the infimum in $\alpha$ (in (25)) does not yield in general a unique minimum. Formally, the infimum in $\alpha$ yields the optimal hedging strategy.... When there exists a unique optimal $\alpha$ that is smooth in $(X, t)$, the hedge $\alpha_{t}=\alpha\left(X_{t}, t\right)$ leads to the following price dynamics

$$
\mathrm{d} S_{t}=\sigma\left(S_{t}\right) \mathrm{d} B_{t}+k \mathrm{~d} \alpha_{t}, \quad \mathrm{~d} X_{t}=\sigma\left(S_{t}\right) \mathrm{d} B_{t}
$$

and thus we have

$$
\left\{\begin{array}{l}
\mathrm{d} S_{t}=\sigma\left(S_{t}\right)\left(1+k \frac{\partial \alpha}{\partial X}\left(X_{t}, t\right)\right) \mathrm{d} B_{t}+b_{t} \mathrm{~d} t, \\
\mathrm{~d} X_{t}=\sigma\left(S_{t}\right) \mathrm{d} B_{t},
\end{array}\right.
$$

for some drift $b_{t}\left(=\frac{\partial \alpha}{\partial t}\left(X_{t}, t\right)+\frac{1}{2} \sigma^{2}\left(S_{t}\right) \frac{\partial^{2} \alpha}{\partial X^{2}}\left(X_{t}, t\right)\right)$. Finally, let us remark that if we assume that $\sigma$ and $\Phi$ are of class $C_{b}^{1,1}$ then, if $\frac{k}{\lambda}\left\|\sigma^{\prime}\right\|_{\infty}$ is small enough, there exists a unique such optimal strategy (which is smooth in $\left.X, t\right) \ldots$. 
Remark 3.3. It is possible to adapt the above results to the case when we incorporate a drift term in (1). Although somewhat more technical, the approach is exactly the same and the computations are straightforward. Let us only mention here that Corollary 3.1 remains valid in the case when we replace $\sigma \mathrm{d} B_{t}$ by $\sigma \mathrm{d} B_{t}+b \mathrm{~d} t$ where $\sigma$ and $b$ are constants. Of course, when we add a drift term, we need to modify the implicit definition (23) of the indifference price: indeed, denoting by $V_{0}$ the same value function as $V$ but with $\Phi \equiv 0$, the indifference price is then defined by

$$
V(C, S, t)=V_{0}(C-\pi, S, t) .
$$

Proof of Theorem 3.1. It is a straightforward application of stochastic control theory and viscosity solutions once we have obtained (21)-(22). Indeed, $V$ solves the HJB equation (20) with $\left.V\right|_{t=0}=U(P-\Phi)$. Next, as is well-known, one looks for a solution $V$ of the following form: $V=1-\mathrm{e}^{-\lambda(P-\pi(X, t))}$. And we obtain (25)!

Proof of Corollary 3.1. One simply observes that (25) reduces to the usual Black-Scholes theory with a unique optimal $\alpha$ given by $\alpha=\partial \pi / \partial X(X, t)$. And (27) follows easily.

Proof of Corollary 3.2. Both statements are formally obvious since Eq. (25) goes to Eq. (28) as $\lambda$ goes to $0_{+}$and to Eq. (29) as $\lambda$ goes to $+\infty$. The justification of these formal asymptotics is a simple application of viscosity solutions theory.

We now explain some asymptotics as $k$ goes to 0 that are valid for an arbitrary utility function (and for the case when we add a drift term to (1)). Using the HJB equations satisfied by $V$, one shows easily, at least formally, that the indifference price satisfies

$$
\pi=\pi_{\mathrm{BS}}(S, t)+\mathrm{O}(k)
$$

(it is in fact possible to make a more precise expansion but we shall not do so here...), where $\pi_{\mathrm{BS}}$ is the Black-Scholes price namely $\pi_{\mathrm{BS}}(S, t)=E\left[\Phi\left(S_{T}\right) \mid S_{t}=S\right]$ where $S_{t}$ solves (1) with $k=0$. This formal argument is once more automatically justified by viscosity solutions arguments - for instance). If we assume that $\sigma \in C_{b}^{1,1}$, then, for $k$ small enough, we have a unique optimal strategy $\alpha$ (see also Remark 3.2) and we deduce easily that we have as $k$ goes to $0_{+}$

$$
\alpha=\frac{\partial \pi_{\mathrm{BS}}}{\partial X}(X, t)+\mathrm{O}(k)=\frac{\partial \pi_{\mathrm{BS}}}{\partial S}(S, t)+\mathrm{O}(k) .
$$

Finally, this leads to the following dynamics (or effective dynamics) for $S_{t}$ namely

$$
\mathrm{d} S_{t}=\sigma\left(S_{t}\right)\left(1+k \frac{\partial^{2} \pi_{\mathrm{BS}}}{\partial S^{2}}\left(S_{t}, t\right)+\mathrm{O}\left(k^{2}\right)\right) \mathrm{d} B_{t}+\tilde{b}_{t} \mathrm{~d} t
$$

for some $\tilde{b}_{t}$ that we do not detail here. In other words, the resulting volatility (that we may call effective volatility) is given by

$$
\sigma_{\mathrm{eff}}(S)=\sigma(S)\left\{1+k \frac{\partial^{2} \pi_{\mathrm{BS}}}{\partial S^{2}}(S, t)+\mathrm{O}\left(k^{2}\right)\right\} .
$$

Let us also mention that it is possible to obtain higher order terms in an asymptotic expansion in $k$.

Remark 3.4. Let us observe that everything we did above (and in the preceding section) extends mutatis mutandis to situations where $\sigma$ depends also on $t(\sigma=\sigma(S, t))$, and where the pay-off is of the form $\int_{t}^{\mathrm{T}} \Phi\left(s, S_{S}\right) \mathrm{d} s+\Phi\left(S_{T}\right)$.

A closely related asymptotic result holds when we consider a "small" pay-off of typical size $\epsilon$. In other words, we consider $\epsilon \Phi$ instead of $\Phi$. Then, in order to retain some risk aversion, we scale accordingly the utility function replacing $U(z)$ by $U\left(\frac{z}{\epsilon}\right)$. We may now perform a simple scaling analysis that shows that the indifference price may be written as $\epsilon \pi$, the control $\alpha_{t}$ as $\epsilon \alpha_{t}$ and thus this amounts to replace $k$ by $\epsilon k$ and (31)-(34) remain true replacing $k$ by $k \epsilon$. In particular, we have

$$
\sigma_{\mathrm{eff}}=\sigma\left\{1+k \epsilon \frac{\partial^{2} \pi_{\mathrm{BS}}}{\partial S^{2}}+\mathrm{O}\left(\epsilon^{2}\right)\right\}
$$


We now conclude this section by explaining the modifications in the above results when we use ( $\left.1^{\prime}\right)$ instead of (1). The reductions made above lead to (21) with

$$
\left\{\begin{array}{l}
\mathrm{d} X_{s}=\sigma\left(\mathrm{e}^{k \alpha_{s}} X_{S}\right) X_{s} \mathrm{~d} B_{s}, \\
\mathrm{~d} P_{s}=\frac{\mathrm{e}^{k \alpha_{s}}-1}{k} X_{s} \sigma\left(\mathrm{e}^{k \alpha_{s}} X_{S}\right) \mathrm{d} B_{S}=\frac{\mathrm{e}^{k \alpha_{s}}-1}{k} \mathrm{~d} X_{s} .
\end{array}\right.
$$

The first modification we notice is that the quantity that plays the role of the hedge in Black-Scholes theory is now $\beta=\left(\mathrm{e}^{k \alpha}-1\right) / k$ while $\alpha$ is really the hedge and that, necessarily, we have $\beta \geqslant-1 / k$.

Then, Theorem 3.1 is still true provided we replace (25) by

$$
\left\{\begin{array}{l}
\frac{\partial \pi}{\partial t}+\frac{1}{2} \inf _{\alpha \in \mathbb{R}}\left\{\sigma^{2}\left(\mathrm{e}^{k \alpha} X\right) X^{2}\left[\frac{\partial^{2} \pi}{\partial X^{2}}+\lambda\left(\frac{\mathrm{e}^{k \alpha}-1}{k}-\frac{\partial \pi}{\partial x}\right)^{2}\right]\right\}=0 \\
\text { or } \\
\frac{\partial \pi}{\partial t}+\frac{1}{2} \inf _{\beta \geqslant-1 / k}\left\{\sigma^{2}((1+k \beta) X) X^{2}\left[\frac{\partial^{2} \pi}{\partial X^{2}}+\lambda\left(\beta-\frac{\partial \pi}{\partial x}\right)^{2}\right]\right\}=0 .
\end{array}\right.
$$

In particular, if $\sigma$ is independent of $S$, the equation reduces to

$$
\frac{\partial \pi}{\partial t}+\frac{1}{2} \sigma^{2} X^{2}\left\{\frac{\partial^{2} \pi}{\partial X^{2}}+\lambda\left(\frac{\partial \pi}{\partial X}+\frac{1}{k}\right)_{+}^{2}\right\}=0,
$$

an equation that remains nonlinear in general because of the constraint $(\beta \geqslant-1 / k)$. However, if we assume in addition that we have

$$
\Phi^{\prime}(X)>-\frac{1}{k} \text { for all } X \geqslant 0
$$

then, because of the strict maximum principle, the indifference price is simply given by the Black-Scholes price $\pi_{\mathrm{BS}}$ that is the solution of

$$
\frac{\partial \pi_{\mathrm{BS}}}{\partial t}+\frac{1}{2} \sigma^{2} X^{2} \frac{\partial^{2} \pi_{\mathrm{BS}}}{\partial X^{2}}=0
$$

with $\left.\pi_{\mathrm{BS}}\right|_{t=T}=\Phi$. Indeed, we then have

$$
\frac{\partial \pi_{\mathrm{BS}}}{\partial X}>-\frac{1}{k} \text { for all } X, t<T
$$

and thus $\pi_{\mathrm{BS}}$ solves (38) (or (37)). In addition, the optimal feedback is given by $\left(\mathrm{e}^{k \alpha}-1\right) / k=\partial \pi_{\mathrm{BS}} / \partial X$ or in other words

$$
\alpha=\frac{1}{k} \log \left(1+k \frac{\partial \pi_{\mathrm{BS}}}{\partial X}\right) .
$$

And the effective price dynamics become

$$
\begin{aligned}
\frac{\partial S_{t}}{S_{t}} & =\sigma \mathrm{d} B_{t}+k \mathrm{~d} \alpha_{t} \\
& =\sigma \mathrm{d} B_{t}+\frac{k}{1+k \partial \pi_{\mathrm{BS}} / \partial X} \frac{\partial^{2} \pi_{\mathrm{BS}}}{\partial X^{2}} \mathrm{~d} X_{t}+b_{t}^{1} \mathrm{~d} t
\end{aligned}
$$

or

$$
\frac{\partial S_{t}}{S_{t}}=\sigma\left(1+\frac{k X_{t}}{1+k \frac{\partial \pi_{\mathrm{BS}}}{\partial X}\left(X_{t}, t\right)} \frac{\partial^{2} \pi_{\mathrm{BS}}}{\partial X^{2}}\left(X_{t}, t\right)\right) \mathrm{d} B_{t}+\tilde{b}_{t} \mathrm{~d} t
$$

for some $b_{t}^{1}, \tilde{b}_{t}$ that we do not detail here, and $\mathrm{d} X_{t}=\sigma X_{t} \mathrm{~d} B_{t}$.

As $\lambda$ goes to 0 , the indifference price goes to the solution of

$$
\frac{\partial \pi}{\partial t}+\frac{1}{2} \inf _{y \in \mathbb{R}}\left\{\sigma^{2}(y) X^{2} \frac{\partial^{2} \pi}{\partial X^{2}}\right\}=0
$$


with $\left.\pi\right|_{t=0}=\Phi$.

The limit as $\lambda$ goes to $+\infty$ is much more delicate. We need to consider for all $X \geqslant 0$

$$
\hat{\Phi}(X)=\inf \left\{\tilde{\Phi}(X) \mid \tilde{\Phi} \geqslant \Phi, \frac{\partial \tilde{\Phi}}{\partial X} \geqslant \frac{1}{k} \text { on } \mathbb{R}\right\} ;
$$

and one can show that $\hat{\Phi}$ is given by

$$
\hat{\Phi}(X)=\sup _{X \geqslant Z \geqslant 0}\left\{\Phi(X-Z)-\frac{1}{k} Z\right\} .
$$

Then, as $\lambda$ goes to $+\infty$, the indifference price converges to the solution of

$$
\frac{\partial \pi}{\partial t}+\frac{1}{2} \sigma^{2}\left(\left(1+k \frac{\partial \pi}{\partial X}\right) X\right) X^{2} \frac{\partial^{2} \pi}{\partial X^{2}}=0
$$

with $\left.\pi\right|_{t=0}=\hat{\Phi}$.

We conclude by stating the analogue of (35) namely

$$
\sigma_{\mathrm{eff}}=\sigma\left\{1+k \epsilon S \frac{\partial^{2} \pi_{\mathrm{BS}}}{\partial S^{2}}+\mathrm{O}\left(\epsilon^{2}\right)\right\},
$$

where $\pi_{\mathrm{BS}}$ is the Black-Scholes price namely $\pi_{\mathrm{BS}}(S, t)=E\left[\Phi\left(S_{T}\right) \mid S_{t}=S\right]$ with $S_{t}$ solution of

$$
\frac{\mathrm{d} S_{t}}{S_{t}}=\sigma\left(S_{t}\right) \mathrm{d} B_{t}
$$

\section{Variants and extensions}

We mention briefly in this section some variants and extensions of the preceding results in several directions namely (i) interest rates, (ii) more general influence models and (iii) multidimensional assets.

The first one concerns interest rates and we simply observe that incorporating simple interest rates models is a straightforward matter that creates no additional difficulty and we skip this easy adaptation in order to restrict the length of this paper.

Concerning other influence models, we mention that we studied many other models where one replaces the term $\left(-k u_{t} \mathrm{~d} t\right)$ in $(1)$ or $\left(1^{\prime}\right)$ by other terms of a similar nature. Our general approach can be adapted to those situations and we shall not attempt here to describe numerous possibilities we looked at. For instance, one may incorporate memory or relaxation effects. One may also consider the case when $k$ is no more a constant but a function of $S_{t}$ and possibly even of $\alpha_{t} \ldots$ Once again, the adaptations are straightforward. However, one variant is slightly more delicate to handle namely the case when $k u_{t}$ is replaced by a nonlinear term $k\left(u_{t}\right)$ where $k$ is, for instance, a Lipschitz function on $\mathbb{R}$ such that we have

$$
k(x)-k x \text { is bounded on } \mathbb{R},
$$

where $k \geqslant 0$. An example of such a $k$ is provided by

$$
k(x)=0 \quad \text { if } x \in[-a, b], \quad k(x)=k(x-b) \quad \text { if } x \geqslant b, \quad k(x)=k(x+a) \quad \text { if } x \leqslant-a,
$$

where $k \geqslant 0, a \geqslant 0, b \geqslant 0$, example that corresponds to an influence which is perceptible only when the rate of buying or selling orders exceeds some given threshold. In such a nonlinear situation, one has to adapt the results (and methods of proofs of [12] and we briefly sketch here, at a formal level, how this can be done "in the case of (1)" that is

$$
\mathrm{d} S_{t}=\sigma\left(S_{t}\right) \mathrm{d} B_{t}-k\left(u_{t}\right) \mathrm{d} t .
$$

As we did in Section 2, we introduce $X_{t}=S_{t}-k \alpha_{t}$ and $P_{t}=C_{t}+\alpha_{t} S_{t}-k \alpha_{t}^{2} / 2$ and we have

$$
\left\{\begin{array}{l}
\mathrm{d} X_{t}=\sigma\left(X_{t}+k \alpha_{t}\right) \mathrm{d} B_{t}-\ell\left(u_{t}\right) \mathrm{d} t, \\
\mathrm{~d} P_{t}=\alpha_{t} \sigma\left(X_{t}+k \alpha_{t}\right) \mathrm{d} B_{t}-\alpha_{t} \ell\left(u_{t}\right) \mathrm{d} t=\alpha_{t} \mathrm{~d} X_{t}
\end{array}\right.
$$


with $\ell(u)=k(u)-k u$ on $\mathbb{R}$. And one can show that the value function is equal (for all $S, \alpha, t<T$ ) to

$$
V(X, P, t)=\sup _{\alpha_{t}, u_{t} \text { adapted }} E\left[U\left(P_{T}-\Phi\left(X_{T}\right)\right) \mid P_{t}=P, X_{t}=X\right]
$$

which solves the following HJB equation

$$
\frac{\partial V}{\partial t}+\sup _{\alpha, u}\left\{\frac{1}{2} \sigma^{2}(X+k x)\left[\frac{\partial^{2} V}{\partial X^{2}}+\alpha^{2} \frac{\partial^{2} V}{\partial P^{2}}+2 \alpha \frac{\partial^{2} V}{\partial X \partial t}\right]-\ell(u) \frac{\partial V}{\partial X}-\alpha \ell(u) \frac{\partial V}{\partial P}\right\}=0 \quad \text { for all } X, t<T
$$

with $\left.V\right|_{t=T}=U(P-\phi(X))$. At this stage, it is then straightforward to adapt the results obtained in the preceding section. For instance, if $\sigma$ is constant, $\Phi \equiv 0$ ("fair value") and $U=1-\mathrm{e}^{-\lambda z}$, then we have

$$
V=U\left(C+\alpha S-\frac{k}{2} \alpha^{2}+\frac{L^{2}}{\lambda \sigma^{2}}(T-t)\right)
$$

where $L=\sup _{u \in \mathbb{R}}|\ell(u)|$.

Our final extension concerns situations involving many assets (or a multidimensional one...) that is

$$
\begin{aligned}
\mathrm{d} S_{t} & =\sigma\left(S_{t}\right) \cdot \mathrm{d} B_{t}-K \cdot u_{t} \mathrm{~d} t, \\
\mathrm{~d} \alpha_{t} & =-u_{t} \mathrm{~d} t, \\
\mathrm{~d} C_{t} & =u_{t} \cdot S_{t} \mathrm{~d} t,
\end{aligned}
$$

where $S_{t} \in \mathbb{R}^{N}, \sigma$ is a Lipschitz $N \times m$ matrix such that $\sigma \sigma^{\mathrm{T}}$ is bounded and positive (uniformly on $\mathbb{R}^{N}$ ), $B_{t}$ is a standard $m$-dimensional Brownian matrix, $\alpha_{t} \in \mathbb{R}^{N}$ and $K$ is a $N \times N$ matrix (not necessarily diagonal).

We emphasize the fact that the "influence" matrix $K$ is not necessarily diagonal, a fact which means that buying or selling significant quantities of one of the assets may influence the price of the others (or some of the others...).

One can then follow and adapt trivially all the arguments made in the previous sections and the results obtained above are extended in a very natural way to this multidimensional setting. For instance, the value of a quantity $\alpha$ of the assets $S$ is

$$
\alpha \cdot S-\frac{1}{2} K \alpha \cdot \alpha
$$

(or $\left(1-\mathrm{e}^{-K_{i j} \mu_{j}}\right) /\left(K_{i k} \mu_{k}\right) \mu_{i}$ if $\mathrm{d} S_{t}^{i}$ in (49) is replaced by $\left.\mathrm{d} S_{t}^{i} / S_{t}^{i} \ldots\right)$,

and the analogue of the asymptotic formula (35) is given by

$$
\sigma_{\text {eff }}=\left(I+\epsilon K \cdot \frac{\partial^{2} \pi_{\mathrm{BS}}}{\partial S^{2}}+\mathrm{O}\left(\epsilon^{2}\right)\right) \cdot \sigma .
$$

Let us observe finally that, even if we consider assets that are a priori independent and a diagonal matrix $K$, our results show that hedging an option whose pay-off depends upon all the assets will create correlations between those assets.

\section{References}

[1] M. Avellaneda, M.D. Lipkin, A market induced mechanism for stock pinning, Preprint.

[2] K. Back, Insider trading in continuous time, Review of Financial Studies 5 (1992) 387-409.

[3] K. Back, C.H. Cao, G. Willard, Imperfect condition among informed traders, J. Finance LV (2000) 2117-2155.

[4] M. Bardi, I. Capuzzo-Dolcetta, Optimal Control and Viscosity Solutions of Hamilton-Jacobi-Bellman Equations, Birkhäuser, Boston, 1997.

[5] F. Black, M. Scholes, The pricing of options and corporate liabilities, J. Political Economy 81 (1973) 637-659.

[6] G. Constantinides, Th. Zariphopoulou, Bounds on prices of continnent claims in an intertemporal economy with proportional transaction costs and general preferences, Finance Stoch. 3 (1999) 345-369.

[7] W.H. Fleming, M.H. Soner, Controlled Markow Processes and Viscosity Solutions, Springer, Berlin, 1993.

[8] H. Föllmer, Stock price fluctuation as a diffusion in a random environment, in: S.D. Howison, F.P. Kelly, P. Wilmott (Eds.), Mathematical Models in Finance, Chapman \& Hall, London, 1995.

[9] R. Frey, A. Stremme, Portfolio insurance and volatility, Department of Economics, Univ. of Bonn, Discussion paper B-256.

[10] A.S. Kyle, Continuous auctions and insider trading, Econometrica 53 (1985) 1315-1335.

[11] J.-M. Lasry, P.-L. Lions, Towards a self-consistent theory of volatility, Preprint.

[12] J.-M. Lasry, P.-L. Lions, Une classe nouvelle de problèmes singuliers de contrôle stochastique, C. R. Acad. Sci. Paris, Ser. I 331 (2000) 879-885. 
[13] G. Lasserre, Asymmetric information and imperfect competition in a continuous time multivariate security model, Finance and Stochastics 8 (2004) 285-309.

[14] R. Merton, Theory of rational option pricing, Bull. J. Econom. Manag. Sci. 4 (1973) 141-183.

[15] M. Musiela, Th. Zariphopoulou, Indifference prices and related measures, Preprint. 